\title{
Association of TCF7L2 polymorphisms with susceptibility to type 2 diabetes in 4,087 Japanese subjects
}

\author{
Kazuaki Miyake $\cdot$ Yukio Horikawa $\cdot$ Kazuo Hara $\cdot$ Kazuki Yasuda $\cdot$ Haruhiko Osawa \\ Hiroto Furuta · Yushi Hirota $\cdot$ Kazuya Yamagata $\cdot$ Yoshinori Hinokio $\cdot$ Yoshitomo Oka • \\ Naoko Iwasaki · Yasuhiko Iwamoto · Yuichiro Yamada · Yutaka Seino · Hiroshi Maegawa · \\ Atsunori Kashiwagi $\cdot$ Ken Yamamoto $\cdot$ Katsushi Tokunaga $\cdot$ Jun Takeda $\cdot$ Hideichi Makino · \\ Kishio Nanjo · Takashi Kadowaki · Masato Kasuga
}

Received: 16 August 2007/ Accepted: 16 November 2007/Published online: 21 December 2007

(C) The Japan Society of Human Genetics and Springer 2007

\begin{abstract}
Transcription factor 7-like 2 (TCF7L2) has been shown to be associated with type 2 diabetes mellitus in multiple ethnic groups. Regarding the Asian population, Horikoshi et al. (Diabetologia 50:747-751, 2007) and Hayashi et al. (Diabetologia 50:980-984, 2007) reported that single nucleotide polymorphisms (SNPs) in TCF7L2 were associated with type 2 diabetes in the Japanese
\end{abstract}

Electronic supplementary material The online version of this article (doi:10.1007/s10038-007-0231-5) contains supplementary material, which is available to authorized users.

K. Miyake · Y. Hirota $\cdot$ M. Kasuga $(\bowtie)$

Division of Diabetes, Metabolism and Endocrinology, Department of Internal Medicine, Kobe University Graduate

School of Medicine, 7-5-1 Kusunoki-cho, Chuo-ku,

Kobe 650-0017, Japan

e-mail: kasuga@med.kobe-u.ac.jp

Y. Horikawa $\cdot$ J. Takeda

Department of Diabetes and Endocrinology Division

of Molecule and Structure, Gifu University School

of Medicine, Gifu, Japan

Y. Horikawa

Laboratory of Medical Genomics, Biosignal Genome Resource

Center, Institute for Molecular and Cellular Regulation,

Gunma University, Maebashi, Japan

\section{K. Hara · T. Kadowaki}

Department of Metabolic Diseases, Graduate School

of Medicine, University of Tokyo, Tokyo, Japan

\section{K. Yasuda}

Department of Metabolic Disorder, Research Institute,

International Medical Center of Japan, Tokyo, Japan

H. Osawa $\cdot$ H. Makino

Department of Molecular and Genetic Medicine, Ehime

University Graduate School of Medicine, Ehime, Japan population, while contradictory results were reported for Han Chinese populations. The aim of this study was to investigate the associations of the TCF $7 L 2$ gene with type 2 diabetes using a relatively large sample size: 2,214 Japanese individuals with type 2 diabetes and 1,873 normal controls. The minor alleles of rs7903146, rs11196205, and rs12255372 showed significant associations with type 2

\section{H. Furuta $\cdot$ K. Nanjo}

The First Department of Medicine, Wakayama Medical University, Wakayama, Japan

K. Yamagata

Department of Metabolic Medicine, Graduate School

of Medicine, Osaka University, Osaka, Japan

Y. Hinokio $\cdot$ Y. Oka

Division of Molecular Metabolism and Diabetes, Tohoku

University Graduate School of Medicine, Sendai, Japan

N. Iwasaki · Y. Iwamoto

Department of Medicine, Diabetes Center,

Tokyo Women's Medical University, Tokyo, Japan

Present Address:

Y. Yamada

Department of Internal Medicine, Akita University School

of Medicine, Akita, Japan

Y. Seino

Kansai Electric Power Hospital, Osaka, Japan

H. Maegawa · A. Kashiwagi

Division of Endocrinology and Metabolism Department

of Medicine, Shiga University of Medical Science, Shiga, Japan

K. Yamamoto

Department of Molecular Genetics, Medical Institute

of Bioregulation, Kyushu University, Fukuoka, Japan 
Table 1 Clinical characteristics of each sample set. Data are means \pm SD. BMI Body mass index

\begin{tabular}{|c|c|c|c|c|c|c|}
\hline & \multicolumn{2}{|l|}{ Kobe } & \multicolumn{2}{|l|}{ Gunma } & \multicolumn{2}{|l|}{ Consortium } \\
\hline & Diabetes & Control & Diabetes & Control & Diabetes & Control \\
\hline$n$ & 465 & 323 & 576 & 576 & 1,173 & 974 \\
\hline Male participants $(\%)$ & 59.6 & 45.8 & 56.1 & 40.4 & 56.6 & 43.1 \\
\hline Age at study (years) & $60.5 \pm 10.7$ & $75.6 \pm 8.1$ & $60.2 \pm 11.5$ & $67.3 \pm 6.5$ & $62.5 \pm 8.8$ & $69.2 \pm 7.0$ \\
\hline BMI & $24.3 \pm 3.9$ & $21.4 \pm 3.5$ & $23.9 \pm 4.2$ & $23.0 \pm 2.9$ & $23.1 \pm 2.9$ & $22.6 \pm 3.0$ \\
\hline $\mathrm{HbA}_{1 \mathrm{c}}(\%)$ & $8.1 \pm 2.0$ & $5.0 \pm 0.4$ & $7.8 \pm 3.5$ & $5.0 \pm 0.4$ & $7.5 \pm 1.5$ & $4.9 \pm 0.4$ \\
\hline
\end{tabular}

diabetes $\left(\mathrm{OR}=1.48, P=2.7 \times 10^{-4} ; \mathrm{OR}=1.39, P=\right.$ $4.6 \times 10^{-4} ; \mathrm{OR}=1.70, P=9.8 \times 10^{-5}$, respectively) in the combined sample sets. However, neither rs11196218 nor rs290487 showed a significant association. These results indicate that TCF7L2 is an important susceptibility gene for type 2 diabetes in the Japanese population.

Keywords Type 2 diabetes $\cdot$ Polymorphism $\cdot \beta$-cell function - Transcription factor 7-like 2 (TCF7L2) · Association study

\section{Introduction}

The transcription factor 7-like 2 gene (TCF7L2) is one of the most convincing susceptibility genes for type 2 diabetes. Following the initial report (Grant et al. 2006), there have been a number of association studies in various ethnic groups (Florez et al. 2006; Zhang et al. 2006; Saxena et al. 2006). Regarding the Asian population, Horikoshi et al. (2007) reported that a single nucleotide polymorphism (SNP), rs7903146, in TCF7L2 is associated with type 2 diabetes in the Japanese population but that other SNPs (rs7895340, rs11196205, rs12255372) are not. The minor allele frequencies of these SNPs in Japanese were also found to be much lower than those of Caucasians. Hayashi et al. (2007) replicated the association of TCF7L2 with type 2 diabetes in Japanese. Contradictory results were reported for Han Chinese populations ( $\mathrm{Ng}$ et al. 2007; Chang et al. 2007), but these two reports found that other common SNPs (rs11196218 and rs290487, respectively) were associated with type 2 diabetes. This apparent difference between Asian populations could be due to the relatively small sample sizes involved. Recently, variants

\section{K. Tokunaga}

Department of Human Genetics, Graduate School of Medicine,

University of Tokyo, Tokyo, Japan

Y. Yamada · Y. Seino

Department of Diabetes and Clinical Nutrition,

Kyoto University School of Medicine, Kyoto, Japan in the TCF7L2 gene also were reported to be associated with $\beta$-cell function (Schäfer et al. 2007; Lyssenko et al. 2007) and response to sulfonylureas in Caucasians (Pearson et al. 2007). To clarify the association of the TCF7L2 gene with type 2 diabetes and $\beta$-cell function in an Asian population, we have performed association studies using a relatively large Japanese sample set: 2,214 Japanese individuals with type 2 diabetes and 1,873 normal controls.

\section{Subjects and methods}

\section{Subjects}

Three sample sets were involved. The Kobe set and the Gunma set samples were recruited from hospitals in Hyogo and Gunma prefecture, respectively. The Consortium set samples were recruited from seven districts in Japan by the Study Group of the Millennium Genome Project for Diabetes Mellitus. The Kobe, Gunma, and Consortium sets were independent of one another. The inclusion criteria for normal, control subjects of the Consortium set were as follows: (1) >60 years of age; (2) $\mathrm{HbA}_{1 \mathrm{c}}$ values $<5.8 \%$; and (3) no family history of type 2 diabetes in first- or second-degree relatives. In the Kobe and Gunma control samples, the inclusion criteria were (1) no past history of diabetes and (2) $\mathrm{HbA}_{1 \mathrm{c}}$ values $<5.8 \%$. The control subjects were hospital patients for annual medical checkup or unrelated disorders. Type 2 diabetes was diagnosed in accordance with WHO criteria. Other forms of diabetes were excluded based on the clinical data. The clinical and laboratory characteristics of the study subjects are shown in Table 1. Written, informed consent was obtained from all participants. The study was approved by the ethics committee of each participating institute.

\section{Genotyping}

Five SNPs (rs7903146, rs11196205, rs12255372, rs11196218, rs290487) were genotyped using TaqMan SNP Genotyping Assays (Applied Biosystems, Foster City, 
CA) or SSP-FCS (sequence specific primer-fluorescence correlation spectroscopy) Assays (Bannai et al. 2004). Of the original five SNPs (rs7903146, rs11196205, rs12255372, rs7901695, rs7895340) in the first report (Grant et al. 2006), we selected three SNPs (rs7903146, rs11196205, rs12255372) for the following reasons: the original five SNPs are located in one linkage disequilibrium (LD) block surrounding exon 4 in the Japanese population (Supplementary Figure 1), which is similar to the case in Caucasians (Grant et al. 2006); rs7901695 and rs7895340 are in almost complete LD with rs7903146 $\left(r^{2}=1\right)$ and rs11196205 $\left(r^{2}=0.90\right)$, respectively, in the Japanese population (Horikoshi et al. 2007); there is no common (minor allele frequency $>10 \%$ ) SNP in this LD block (HapMap JPT data). We also genotyped rs11196218 and rs290487, which were associated with type 2 diabetes in Han Chinese, to replicate this association in Japanese. To evaluate our genotyping, 180 samples in the Consortium set were genotyped by both TaqMan SNP Genotyping Assays and SSP-FCS Assays. The concordance rate between these two assays was 100\%: genotypes determined by TaqMan or SSP-FCS methods were identical to those determined by direct sequencing for 48 samples.

The genotyping success rates in the three sample sets were all $>93 \%$. All five SNPs were in Hardy-Weinberg equilibrium (HWE; $P>0.05$ in the Exact test) in both case and control groups of all sample sets.

\section{Clinical assessment}

The clinical profile of each subject was directly determined at the time of entry. HOMA-IR and HOMA- $\beta$ were calculated as follows: HOMA-IR $=$ (fasting insulin $[\mathrm{pmol} / \mathrm{l}]) \times$ glucose $[\mathrm{mmol} / \mathrm{l}]) / 22.5 \times 6$ and HOMA$\beta=$ (fasting insulin $[\mathrm{pmol} / \mathrm{l}]) \times 2) /(\mathrm{glucose} \quad[\mathrm{mmol} / \mathrm{l}]$ $-3.5) \times 6$. Diabetic subjects treated with insulin were excluded from analysis of HOMA-IR and HOMA- $\beta$. Assessments were performed with the combined three sample sets. Data are expressed as means \pm SD.

\section{Statistical analysis}

The differences for SNPs or estimated haplotypes between type 2 diabetic and non-diabetic subjects were compared using Chi-square test under an allelic model. We also performed multiple logistic regression analysis adjusted for age, sex, and BMI under a dominant model. Statistical analysis was performed with the Stat-View program (version 5.0-J; SAS Institute, Cary, NC). The relation of the variants in TCF7L2 with BMI and Homeostasis model assessment (HOMA-IR and HOMA- $\beta$ ) by $t$ test under the dominant model for each SNP was then assessed. The HOMA-IR and HOMA- $\beta$ data were log-transformed for normality. LD and haplotype analyses were performed with SNPAlyze version 5.1 pro software (Dynacom, Mobara, Japan). We considered statistical significance at $P$ values of $<0.01$ and $<0.017$ in the association study for SNPs and for clinical parameters, respectively, after Bonferroni correction. The prevalence of type 2 diabetes in the Japanese population was assumed to be 0.07 . Population attributable risk (PAR) was calculated as PAR $=p(\mathrm{RR}-1) /$ $[p(\mathrm{RR}-1)+1]$, where $p$ and $\mathrm{RR}$ are the risk allele frequency in the general population and the relative risk, respectively, estimated by the prevalence. When the frequency of risk allele, OR, and type I error probability are assumed to be 0.03 (Horikoshi et al. 2007), 1.46 (Cauchi et al. 2007), and 0.05, respectively, based upon the previous study, the power of our combined samples $(2,214$ cases and 1,873 controls) to detect association between SNP rs7903146 and type 2 diabetes is 0.92 . In the case of OR assumed to be 1.69 (Horikoshi et al. 2007), the power of our study is 0.99 .

\section{Results}

We performed association analyses using three independent sample sets. Regarding three SNPs (rs7903146, rs11196205, and rs12255372), which originally showed association with type 2 diabetes, the minor alleles showed a trend toward association with type 2 diabetes in the Kobe set. These SNPs also showed a marginally significant association in the Gunma set and in the Consortium set when multiple testing was considered. In the combined three sample sets (Combined set), the minor alleles of rs7903146, rs11196205, and rs12255372 showed a significant association with susceptibility to the disease $\left(\mathrm{OR}=1.48, \quad P=2.7 \times 10^{-4}\right.$; $\mathrm{OR}=1.39, P=4.6 \times 10^{-4} ; \mathrm{OR}=1.70, P=9.8 \times 10^{-5}$, respectively). These associations remained significant after adjustment for age, sex, and BMI (Table 2). As in a previous report (Horikoshi et al. 2007), the MAF and PAR in our study were much lower (MAF: 0.022-0.072, PAR: $\sim 0.02$ in the Combined set) than those in Caucasians. Neither rs11196218 nor rs290487 showed a significant association in any sample set (Table 2).

LD among the five SNPs in 974 control subjects in the Consortium set was then analyzed. The $D^{\prime}$ and $r^{2}$ values are shown in Table 3. As reported previously for Japanese, three SNPs (rs7903146, rs11196205, and rs12255372) were found to be in modest to strong $\operatorname{LD}\left(D^{\prime}=0.56-1.0\right)$. Haplotypes then were constructed with these SNPs in the Combined set and assessed for association with type 2 diabetes. A haplotype comprising the risk allele of each 


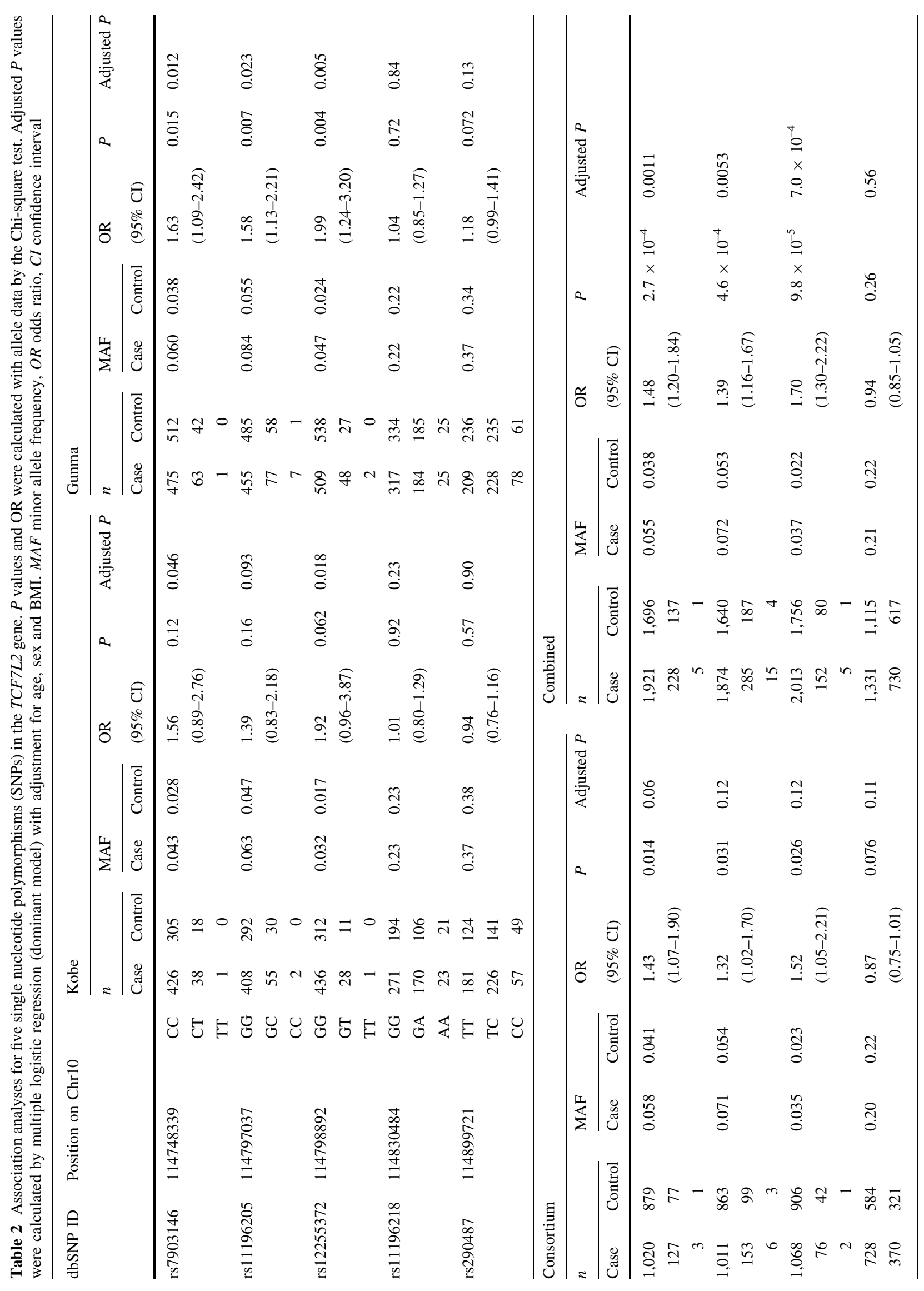




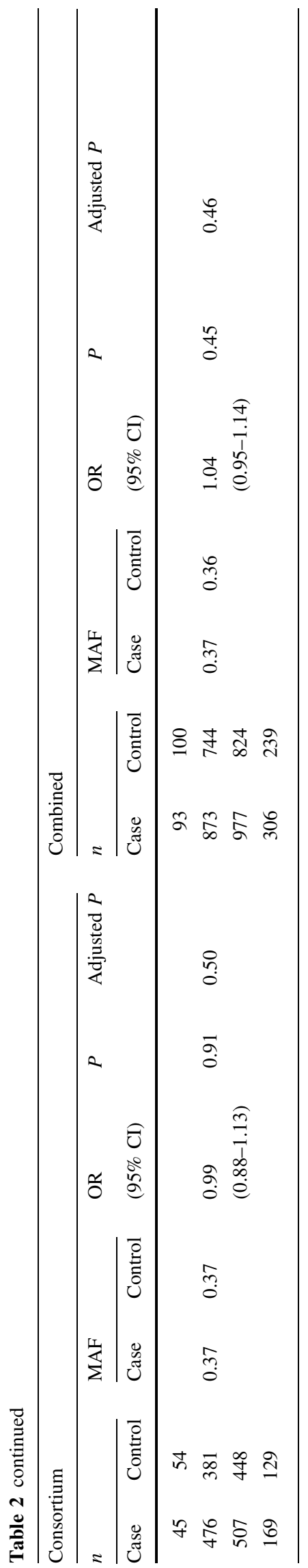

SNP, T-C-T, was significantly associated with type 2 diabetes $\left(P=5.3 \times 10^{-5}\right)$ (Table 4$)$.

The relation of rs 7903146 , rs 11196205 , and rs 12255372 to BMI, HOMA-IR, and HOMA- $\beta$ in the combined cases and controls were then compared. There was no association with BMI in cases or controls. The risk allele of rs7903146 was associated with lower HOMA- $\beta$ (CC $(n=789)$ versus $\mathrm{CT} / \mathrm{TT}(n=83) ; 52.0 \pm 87.6$ versus $35.7 \pm 35.9, P=0.009)$ and lower HOMA-IR (CC vs. CT/TT; $3.2 \pm 4.5$ vs. $2.2 \pm 1.6, P=0.01)$ in the combined diabetic subjects. However, these associations disappeared after adjustment for age, sex, and BMI. No association was found for HOMA- $\beta$ or HOMA-IR in the combined control subjects.

\section{Discussion}

We have found that three SNPs (rs7903146, rs11196205, rs12255372) of TCF7L2 are associated with susceptibility to type 2 diabetes in the Japanese population. Our results are consistent with previous reports for Japanese populations (Horikoshi et al. 2007; Hayashi et al. 2007), but not with other reports for Han Chinese populations ( $\mathrm{Ng}$ et al. 2007; Chang et al. 2007). The apparent difference in the association of these SNPs in Asians could be due to the low frequencies of the SNPs and the relatively small sample sizes used in the previous studies. Since we did not detect any association of rs11196218 or rs290487 in the present study, the associations of the two SNPs in the previous reports for Chinese might be specific to that population. In this study, rs7903146, rs11196205, and rs12255372 were in modest to strong LD. Based on HapMap data (JPT), the LD block surrounding exon 4 of TCF7L2 in Asians does not exceed the gene (Supplementary Figure 1), which is consistent with findings in Caucasians (Grant et al. 2006). Previous reports (Ng et al. 2007; Chang et al. 2007) also found that the three SNPs were in a single LD block while the other two (rs11196218 and rs290487) were not. According to meta-analysis by Cauchi et al. (2007), TCF7L2 is the most reproducible susceptibility gene for type 2 diabetes in various ethnic groups. TCF7L2 also was one of the most significantly associated genes in recent genome-wide association studies (Sladek et al. 2007; WTCCC 2007). While the risk alleles of this gene are not common in East Asians, including Japanese, and the population attributable risk is much lower, TCF7L2 is nevertheless a risk gene for type 2 diabetes in East Asians as well as in other populations. On the other hand, in a very recent online report, polymorphisms in the TCF7L2 gene were found not to be associated with type 2 diabetes in a relatively large study of Pima Indians (Guo et al. 2007). Further investigation is required to 
Table 3 Pairwise linkage disequilibrium (LD) for five SNPs in the TCF7L2 gene. Values of $D^{\prime}$ (left lower) and of $r^{2}$ (upper right) for pairwise LD analysis in 974 control subjects of the Consortium set

\begin{tabular}{llllll}
\hline & rs7903146 & rs11196205 & rs12255372 & rs1196218 & rs290487 \\
\hline rs7903146 & & 0.24 & 0.49 & 0.002 & 0.0036 \\
rs11196205 & 0.56 & & 0.44 & 0.012 & 0.0037 \\
rs12255372 & 0.93 & 1.00 & & 0.007 & 0.0036 \\
rs1196218 & 0.45 & 0.87 & 1.00 & & 0.0002 \\
rs290487 & 0.22 & 0.19 & 0.30 & 0.02 & \\
\hline
\end{tabular}

Table 4 Association analysis for haplotypes with three SNPs (rs7903146, rs11196205, rs12255372). $P$ values were calculated by the chi-square test with estimated haplotype data from the Combined set

\begin{tabular}{llll}
\hline Haplotype & Case & Control & $P$ \\
\hline C-G-G & 0.91 & 0.93 & $1.5 \times 10^{-4}$ \\
C-C-G & 0.032 & 0.031 & 0.72 \\
T-C-T & 0.032 & 0.018 & $5.3 \times 10^{-5}$ \\
T-G-G & 0.020 & 0.017 & 0.30 \\
\hline
\end{tabular}

elucidate the differences in the contribution of the TCF $2 L 2$ gene to type 2 diabetes among various populations.

$T C F 7 L 2$ regulates expression of the proglucagon gene $(G C G)$, which encodes the precursor of glucagon, glucagon-like peptide 1 (GLP-1) (Yi et al. 2005). Several reports have found that polymorphisms of TCF7L2 are associated with $\beta$-cell function (Florez et al. 2006; Saxena et al. 2006; Schäfer et al. 2007; Lyssenko et al. 2007). In this study, the association between the TCF7L2 gene and HOMA- $\beta$ was found to disappear after adjustment for the various factors. Although the relationship of this gene to $\beta$-cell function is not clear in this study, our results suggest that TCF7L2 is an important susceptibility gene for type 2 diabetes in Japanese. The pathophysiological mechanism of this gene in susceptibility to type 2 diabetes remains to be elucidated.

Acknowledgments We are very grateful for Drs. Sumio Sugano and Shoji Tsuji for their contributions and helpful discussions throughout the project. We also thank Ms. Megumi Yamaoka-Sageshima for technical assistance. This work was supported by KAKENHI (Grant-in-Aid for Scientific Research) on Priority Areas "Applied Genomics" and "Comprehensive Genomics" from the Ministry of Education, Culture, Sports, Science and Technology of Japan, and also in part by a New Energy and Industrial Technology Development Organization grant to Y. Horikawa.

\section{References}

Bannai M, Higuchi K, Akesaka T, Furukawa M, Yamaoka M, Sato K, Tokunaga K (2004) Single-nucleotide-polymorphism genotyping for whole-genome-amplified samples using automated fluorescence correlation spectroscopy. Anal Biochem 327:215-221

Cauchi S, El Achhab Y, Choquet H, Dina C, Krempler F, Weitgasser R, Nejjari C, Patsch W, Chikri M, Meyre D, Froguel P (2007)
TCF7L2 is reproducibly associated with type 2 diabetes in various ethnic groups: a global meta-analysis. J Mol Med $85: 777-782$

Chang YC, Chang TJ, Jiang YD, Kuo SS, Lee KC, Chiu KC, Chuang LM (2007) Association study of the genetic polymorphisms of the transcription factor7- like 2 (TCF7L2) gene and type 2 diabetes in the Chinese Population. Diabetes 56:2631-2637

Florez JC, Jablonski KA, Bayley N, Pollin TI, de Bakker PI, Shuldiner AR, Knowler WC, Nathan DM, Altshuler D, Diabetes Prevention Program Research Group (2006) TCF7L2 polymorphisms and progression to diabetes in the Diabetes Prevention Program. N Engl J Med 355:241-250

Grant SF, Thorleifsson G, Reynisdottir I, Benediktsson R, Manolescu A, Sainz J, Helgason A, Stefansson H, Emilsson V, Helgadottir A, Styrkarsdottir U, Magnusson KP, Walters GB, Palsdottir E, Jonsdottir T, Gudmundsdottir T, Gylfason A, Saemundsdottir J, Wilensky RL, Reilly MP, Rader DJ, Bagger Y, Christiansen C, Gudnason V, Sigurdsson G, Thorsteinsdottir U, Gulcher JR, Kong A, Stefansson K (2006) Variant of transcription factor 7-like 2 (TCF7L2) gene confers risk of type 2 diabetes. Nat Genet 38:320-323

Guo T, Hanson RL, Traurig M, Li Muller Y, Ma L, Mack J, Kobes S, Knowler WC, Bogardus C, Baier LJ (2007) TCF7L2 is not a major susceptibility gene for type 2 diabetes in Pima Indians: an analysis of 3501 individuals. Diabetes. [Epub ahead of print]

Hayashi T, Iwamoto Y, Kaku K, Hirose H, Maeda S (2007) Replication study for the association of TCF7L2 with susceptibility to type 2 diabetes in a Japanese population. Diabetologia 50:980-984

Horikoshi M, Hara K, Ito C, Nagai R, Froguel P, Kadowaki T (2007) A genetic variation of the transcription factor 7-like 2 gene is associated with risk of type 2 diabetes in the Japanese population. Diabetologia 50:747-751

Lyssenko V, Lupi R, Marchetti P, Del Guerra S, Orho-Melander M, Almgren P, Sjogren M, Ling C, Eriksson KF, Lethagen AL, Mancarella R, Berglund G, Tuomi T, Nilsson P, Del Prato S, Groop L (2007) Mechanisms by which common variants in the TCF7L2 gene increase risk of type 2 diabetes. J Clin Invest 117:2155-2163

Ng MC, Tam CH, Lam VK, So WY, Ma RC, Chan JC (2007) Replication and identification of novel variants at TCF7L2 associated with type 2 diabetes in Hong Kong Chinese. J Clin Endocrinol Metab 92:3733-3737

Pearson ER, Donnelly LA, Kimber C, Whitley A, Doney AS, McCarthy MI, Hattersley AT, Morris AD, Palmer CN (2007) Variation in TCF7L2 influences therapeutic response to sulfonylureas: a GoDARTs study. Diabetes 56:2178-2182

Saxena R, Gianniny L, Burtt NP, Lyssenko V, Giuducci C, Sjogren $\mathrm{M}$, Florez JC, Almgren $\mathrm{P}$, Isomaa $\mathrm{B}$, Orho-Melander $\mathrm{M}$, Lindblad U, Daly MJ, Tuomi T, Hirschhorn JN, Ardlie KG, Groop LC, Altshuler D (2006) Common single nucleotide polymorphisms in $T C F 7 L 2$ are reproducibly associated with type 2 diabetes and reduce the insulin response to glucose in nondiabetic individuals. Diabetes 55:2890-2895 
Schäfer SA, Tschritter O, Machicao F, Thamer C, Stefan N, Gallwitz B, Holst JJ, Dekker JM, T'hart LM, Nijpels G, van Haeften TW, Haring HU, Fritsche A (2007) Impaired glucagon-like peptide-1induced insulin secretion in carriers of transcription factor 7-like 2 (TCF7L2) gene polymorphisms. Diabetologia 50:2443-2450

Sladek R, Rocheleau G, Rung J, Dina C, Shen L, Serre D, Boutin P, Vincent D, Belisle A, Hadjadj S, Balkau B, Heude B, Charpentier G, Hudson TJ, Montpetit A, Pshezhetsky AV, Prentki M, Posner BI, Balding DJ, Meyre D, Polychronakos C, Froguel P (2007) A genome-wide association study identifies novel risk loci for type 2 diabetes. Nature 445:881-885
Wellcome Trust Case Control Consortium (2007) Genome-wide association study of 14,000 cases of seven common diseases and 3,000 shared controls. Nature 447:661-678

Yi F, Brubaker PL, Jin T (2005) TCF-4 mediates cell typespecific regulation of proglucagon gene expression by betacatenin and glycogen synthase kinase-3beta. J Biol Chem 280:1457-1464

Zhang C, Qi L, Hunter DJ, Meigs JB, Manson JE, van Dam RM, Hu FB (2006) Variant of transcription factor 7-like 2 (TCF7L2) gene and the risk of type 2 diabetes in large cohorts of U.S. women and men. Diabetes 55:2645-2648 